\title{
Conceptual modeling of postmortem evaluation findings to describe dairy cow deaths
}

\author{
C. S. McConnel, ${ }^{11}$ F. B. Garry, ${ }^{*}$ A. E. Hill, ${ }^{\star}$ J. E. Lombard, ${ }^{*} \dagger$ and D. H. Gould ${ }^{*}$ \\ *Integrated Livestock Management, Colorado State University, Fort Collins 80523 \\ †USDA-Animal and Plant Health Inspection Service-Veterinary Services, Centers for Epidemiology and Animal Health, 2150 Centre Ave., \\ Bldg. B, Fort Collins, CO 80526-8117
}

\section{ABSTRACT}

Dairy cow mortality levels in the United States are excessive and increasing over time. To better define cause and effect and combat rising mortality, clearer definitions of the reasons that cows die need to be acquired through thorough necropsy-based postmortem evaluations. The current study focused on organizing information generated from postmortem evaluations into a monitoring system that is based on the fundamentals of conceptual modeling and that will potentially be translatable into on-farm relational databases. This observational study was conducted on 3 highproducing, commercial dairies in northern Colorado. Throughout the study period a thorough postmortem evaluation was performed by veterinarians on cows that died on each dairy. Postmortem data included necropsy findings, life-history features (e.g., birth date, lactation number, lactational and reproductive status), clinical history and treatments, and pertinent aspects of operational management that were subject to change and considered integral to the poor outcome. During this study, 174 postmortem evaluations were performed. Postmortem evaluation results were conceptually modeled to view each death within the context of the web of factors influencing the dairy and the cow. Categories were formulated describing mortality in terms of functional characteristics potentially amenable to easy performance evaluation, management oversight, and research. In total, 21 death categories with 7 category themes were created. Themes included specific disease processes with variable etiologies, failure of disease recognition or treatment, traumatic events, multifactorial failures linked to transition or negative energy balance issues, problems with feed management, miscellaneous events not amenable to prevention or treatment, and undetermined causes. Although postmortem evaluations provide the relevant information necessary for

Received April 15, 2009.

Accepted October 6, 2009.

${ }^{1}$ Corresponding author: craigmcc@colostate.edu framing a cow's death, a restructuring of on-farm databases is needed to integrate this level of detail into useful monitoring systems. Individual operations can focus on combating mortality through the use of employee training related to postmortem evaluations, detailed forms for capturing necropsy particulars and other relevant information related to deaths, and standardized nomenclature and categorization schemes. As much as anything, the simple act of recognizing mortality as a problem might be the most fundamental step toward controlling its progression.

Key words: mortality, necropsy, dairy, cow

\section{INTRODUCTION}

Dairy cow mortality levels in the United States are excessive and increasing over time. Analysis of DHI data $(15,025,035$ lactations in 45,032 herds) demonstrated a $1.6 \%$ increase in death frequency from 1995 to 2005 (Miller et al., 2008). Similarly, the USDAAnimal and Plant Health Inspection Service-Veterinary Services National Animal Health Monitoring System (NAHMS) Dairy 2007 survey reported that $5.7 \%$ of the January 2007 dairy cow inventory died on-farm during 2006, a significant increase from $4.8 \%$ of the January 2002 inventory and $3.8 \%$ of the January 1996 inventory (USDA, 2007b). These numbers are perhaps even more poignant when one considers that this increase in death is occurring even as the age of the US dairy population declines (Hare et al., 2006). This is a growing concern for dairy producers, both because of the obvious economic liability it represents and because of ethical and welfare dimensions (Thomsen and Houe, 2006; NDAWB, 2008).

One might suspect an underlying genetic component to increasing death losses as a result of genetic selection biased toward production indices, with little consideration of animal longevity or disease occurrence. In fact, data suggest that Jersey and crossbred dairy cows have a substantially reduced mortality level relative to purebred Holsteins (G. W. Rogers, Global Technical Advisor, Geno Global Ltd.; personal communication). However, overall favorable genetic trends for survival 
imply that the decline in dairy cow survival is primarily the result of changes in herd management as opposed to genetic selection (Dechow and Goodling, 2008). An exploration of US herd characteristics and practices demonstrated associations between greater levels of mortality and management changes related to intensification (McConnel et al., 2008).

The preponderance of literature investigating dairy cow mortality has analyzed the association between mortality levels and population characteristics such as parity, disease prevalence, or days in lactation rather than describing cow death relative to specific necropsy findings (Thomsen and Houe, 2006; Bar et al., 2008; Dechow and Goodling, 2008; McConnel et al., 2008; Miller et al., 2008). Without necropsy examinations, the recorded cause of death is often determined by producer perceptions (McConnel et al., 2009). Many disease conditions can present with similar clinical abnormalities that when used on their own to categorize causes of death can lead to misclassifications (Loneragan et al., 2001). An important step in defining cause and effect and combating rising mortality lies in more clearly defining the reasons that cows die through a thorough necropsy-based postmortem evaluation.

Recent publications have touted the benefits of and procedures for performing field necropsy examinations (White, 2005; Mason and Madden, 2007; Wagner, 2007). A dead animal that is not evaluated by necropsy is a total economic loss to a producer; however, a thorough necropsy examination can provide valuable management information that could benefit the herd (White, 2005). Although field necropsies can prove laborious, time consuming, and at times fruitless, there are many reasons for performing them. Necropsies are warranted when morbidity or mortality exceed historic or comfortable levels, when there is a perceived treatment failure, for acquiring samples necessary for confirmation of a tentative clinical diagnosis, when presenting signs are dramatic or unusual, or to characterize a disease process when no antemortem observation has been made (Mason and Madden, 2007). Information derived from a necropsy should be viewed in conjunction with background information related to clinical history and treatments to form a thorough postmortem evaluation.

Whereas the costs incurred from necropsies vary depending on who performs the task, the value of a postmortem evaluation is directly related to the accuracy and maintenance of data collected and its application to operational management. It can be difficult to maintain accurate postmortem records that can be easily retrieved to provide valuable insight into historical death patterns and to guide future health planning and programs (White, 2005). Current record systems such as those provided for DHI herds can provide co- pious concrete data regarding life-history features of dead cows but are not configured to facilitate analysis of previous health events that result in a current condition, nor do they assess the cause and effect of various phenomena. In fact, the least-available dairy herd data comprise records of disease and management events and are subject to tremendous variability in the rigor and consistency of their recording (Kelton, 2006). Establishing record system templates that document dairy cow deaths consistently across operations and within the context of historical influences would allow for the easy determination of the number, distribution, and causes of deaths over a period of time and could guide management practices toward the goal of reduced mortality (McConnel et al., 2009).

The current study was founded on the premise that thorough postmortem evaluations could be used to explore causes of dairy cow death not simply as a function of anatomic pathologies but by viewing necropsy findings within the context of historical and environmental influences. Data collection forms have been created that facilitate the capture of concrete data related to the individual animal being evaluated (White, 2005). Such records typically focus on specific life-history features (e.g., birth date, lactation number, lactational and reproductive status), health events, treatments, and necropsy and adjunctive diagnostic findings. Expanding and improving record systems to capture more data related to farm management dynamics can be facilitated through the use of conceptual modeling principles. Modeling provides a foundation for database schemes that prevent redundancy, provide easy entry and retrieval of information, and accommodate new and unexpected items of information (Lescourret et al., 1993). This study focused on organizing information generated from postmortem evaluations into a monitoring system that is based on the fundamentals of conceptual modeling and will potentially be translatable into current on-farm relational databases.

\section{MATERIALS AND METHODS}

\section{The Problem as it Relates to Dairy Complexity}

Necropsy examinations of dead animals to assess and monitor causes of death are rarely performed on US dairies (USDA, 2007a), unlike other intensive livestock management systems, including poultry, swine, and feedlot enterprises (USDA, 2000b) where necropsy monitoring is routine. Dairy industry efforts to effectively decrease mortality losses are thus hampered by a lack of monitoring and information to provide an accurate assessment of the problem. Comparing the dairy and feedlot industries makes it easier to understand the apparent inertia associated with dairy postmor- 
tem evaluations. Within the feedlot industry a small number of observed disease complexes warrant action. Relative to dairy operations, cattle entering feedlots are typically subject to a shorter period within the system and are not faced with the various production challenges of a dairy cow. Demands on feedlot cattle do not include the physiologic challenges associated with pregnancy, parturition, and lactation. Nor are animals in a feedlot operation required to undergo the physical strain of processing (milking, reproductive exams, etc.) multiple times a day as is demanded from milking dairy cattle. As a result, the problems encountered by feedlot cattle are relatively limited and are consequently easier to monitor as compared with the substantially more complex dairy operations.

\section{Feedlot Versus Dairy Mortalities}

The mortality ratio for cattle entering feedlots did not significantly increase from 1994 to 1999 based on the NAHMS Feedlot 1999 survey findings (Loneragan et al., 2001), yet during that same period, the percentage of dead feedlot cattle that had a postmortem examination substantially increased from $45.9 \%$ to $53.9 \%$ (USDA, 2000a). This increase in postmortem examinations was primarily the product of an increase in necropsies performed by nonveterinarians (USDA, 2000a). Most of the feedlot-associated deaths documented in the NAHMS survey resulted from bovine respiratory disease complex (61.2\%), whereas $21.9 \%$ of animals were classified as having died from digestive tract disorders, and $16.9 \%$ died of other disorders (Loneragan et al., 2001).

Conversely, the percentage of dairy cows that die onfarm has significantly increased from 1996 to 2007, yet the NAHMS Dairy 2007 study reported that necropsies were performed on only $13 \%$ of operations $(\sim 9,750$ operations) and only $4.4 \%$ of dead dairy cows $(\sim 23,000$ cows) (USDA, 2007a). Furthermore, the range of dairy producer-attributed causes of death within the Dairy 2007 study included lameness or injury $(20.0 \%)$; mastitis (16.5\%); calving problems $(15.2 \%)$; respiratory problems $(11.3 \%)$; scours, diarrhea, or other digestive problems (10.4\%); other known reasons $(11.6 \%)$; and unknown reasons (15.0\%). The perception is that dairy cows die from a wider range of problems than are typically recognized within the feedlot industry, yet substantially less effort is made in terms of postmortem evaluations to more fully define how and why dairy cows die.

\section{Project Framework}

This observational study was conducted on 3 (A, B, and C) high-producing (13,184, 11,915, and $11,275 \mathrm{~kg}$ of milk/cow per year, respectively), commercial dairies in northern Colorado. Dairy A participated in the study from October 2006 through November 2007, Dairy B participated from November 2006 through September 2007, and dairy C participated from October 2006 through January 2007. Dairies A and C maintained essentially stable inventories of 1,500 and 2,000 Holstein cows (lactating and dry), respectively. Dairy B's inventory of approximately 800 cows consisted of $25 \%$ Jersey and $75 \%$ Holstein cattle (lactating and dry). Lactating cows on dairies $\mathrm{A}$ and $\mathrm{C}$ were predominantly housed year-round in freestall barns with bedding consisting of sand on dairy A and composted manure on dairy C. Lactating cows on dairy B were held in drylots during summer months and freestall barns bedded with sawdust during the winter months. All 3 dairies held dry cows in drylots. Arithmetic mean SCC were 247,698, 198,218, and 219,789 cells/mL for dairies A, B, and $\mathrm{C}$, respectively. Cows were milked 3 times a day on all of the dairies. Approximately $40 \%$ of cows on dairy $\mathrm{A}$ received $\mathrm{bST}$, whereas dairies $\mathrm{B}$ and $\mathrm{C}$ did not use bST. Hoof-care programs on all dairies involved both the treatment of animals observed with lameness and twice-annual maintenance trimming. Nutritional management for all dairies included the use of a TMR and forage testing, with ration formulation by a professional consultant based on production and stage of lactation. The dry period was approximately $55 \mathrm{~d}$ for all dairies. Maternity housing was separate from housing used for other dry cows or lactating cows. On dairies A and B, heifers and mature cows were grouped together within the close-up maternity housing, but on dairy $\mathrm{C}$, heifers and mature cows were grouped separately. On dairies $\mathrm{A}$ and $\mathrm{C}$, the majority of cows calved in a multiple animal area, whereas on dairy $\mathrm{B}$, the majority calved in an individual animal area cleaned after 2 or more calvings. Fresh cows were penned separately from hospital cows, and first-lactation cows were grouped separately from mature cows on all dairies. Approximately 28, 18, and 43 full-time staff participated in milking and cow management activities on dairies $\mathrm{A}, \mathrm{B}$, and $\mathrm{C}$, respectively, with training sessions performed multiple times per year to cover protocols related to milking, calving, and fresh-cow monitoring. Routine veterinary services on dairy A were provided by the Colorado State University College of Veterinary Medicine. Dairies B and $\mathrm{C}$ each employed an in-house veterinarian to provide veterinary services. Operational management on all dairies included the use of on-farm computer systems to track cow- and herd-level data.

\section{Postmortem Evaluations}

Throughout the study period, a thorough postmortem evaluation was performed on most cows that died 


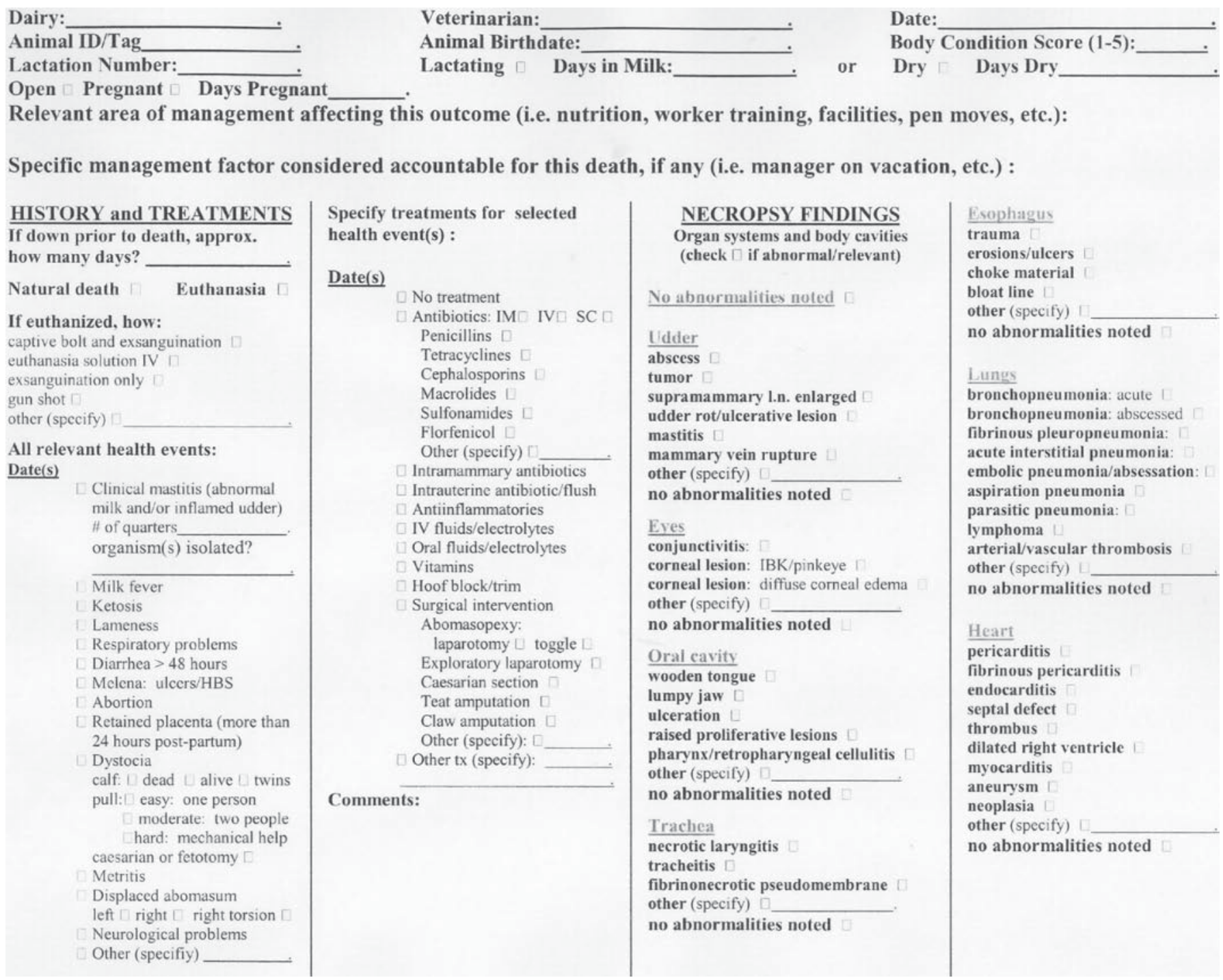

Figure 1A. Postmortem data collection sheets, page 1.

on each dairy. Necropsies were performed on dairy A by one of 2 Colorado State University veterinarians and on dairies $\mathrm{B}$ and $\mathrm{C}$ by the respective in-house veterinarians. All 4 participating veterinarians were trained in standard necropsy techniques (Severidt et al., 2002) via a necropsy protocol overview performed at the Colorado State University Veterinary Diagnostic Laboratory before commencing the project. When possible, the participating veterinarians performed antemortem clinical evaluations on animals to be killed. Necropsy examinations were performed as soon as possible after death and within a maximum of $24 \mathrm{~h}$. If a participating veterinarian could not attend to a cow within $24 \mathrm{~h}$ postmortem, the animal was excluded from the study. The submission of appropriate tissue or biologic samples for further diagnostics was discretionary and based on necropsy findings when additional insight was warranted to confirm or determine the cause of death. Carcasses were disposed of through on-site composting on dairies $\mathrm{A}$ and $\mathrm{C}$ and were removed by a renderer on dairy B.

Results from the postmortem evaluation were recorded in a standardized format (Figure 1A and 1B) that captured concrete data related to specific life-history features (e.g., birth date, lactation number, lactational and reproductive status), health events, treatments, and necropsy findings. No timeframe was set before death for inclusion of events; rather, the veterinarian overseeing the postmortem examination recorded health problems and treatment episodes believed to have potentially influenced the final outcome of death. Most often this included health and treatment events within a current lactation, although data from a previous lactation did occasionally provide useful informa- 


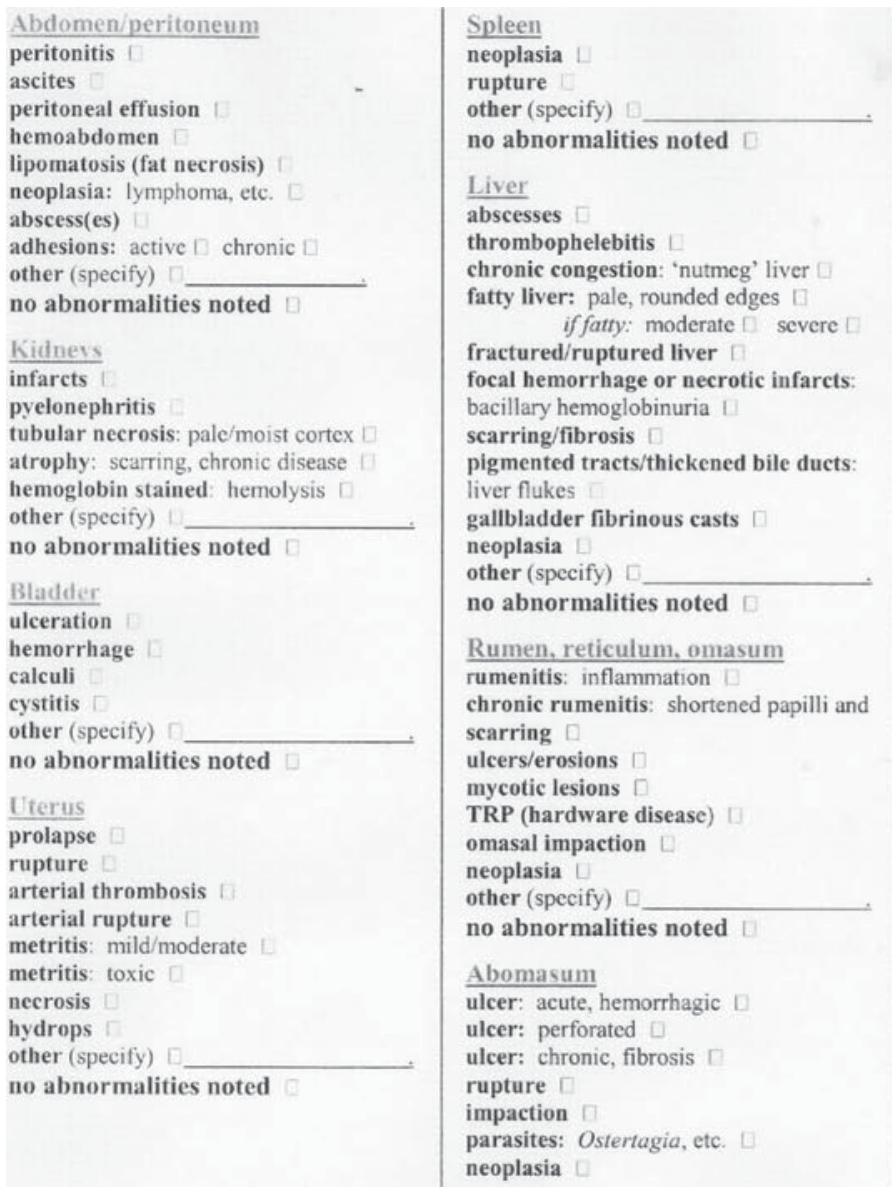

Comments regarding necropsy findings:

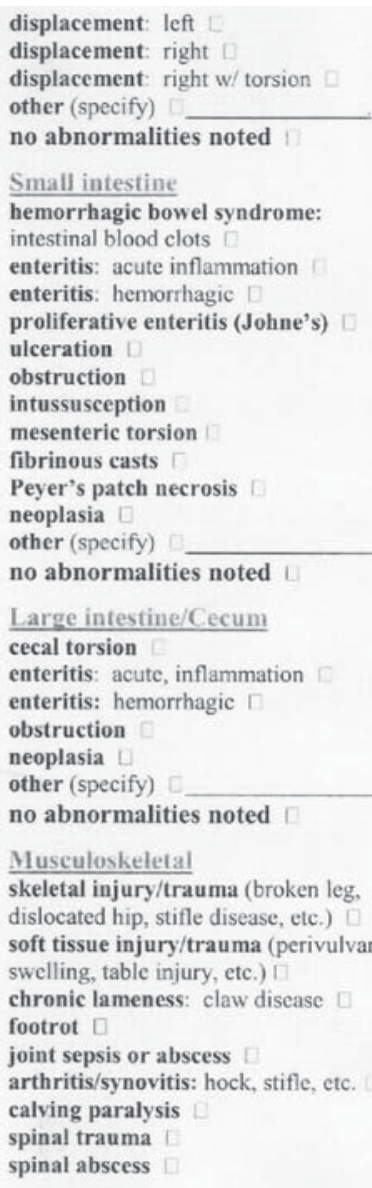

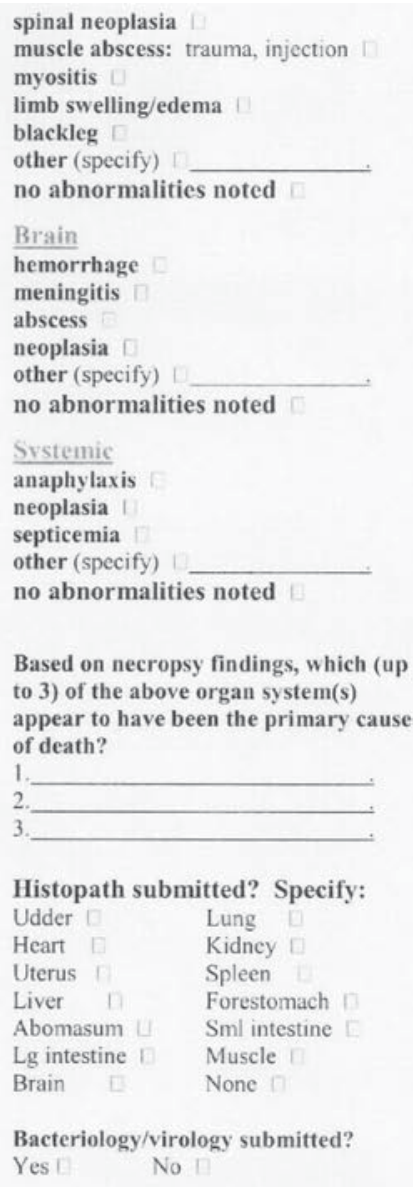

Figure 1B. Postmortem data collection sheets, page 2.

tion related to a death. The participating veterinarians also included more dynamic data inherent to the dairy and believed to be relevant to the death. These data included aspects of operational management that were subject to change and considered integral to the poor outcome. Whereas it was believed that the proximate cause of death could most often be described through necropsy findings, the additional management-oriented information provided a more thorough analysis of any underlying or key determinant causes of death. Borrowing from the language of pathology, this was comparable to establishing a "definitive diagnosis" through the naming of a disease (proximate cause) versus an "etiological diagnosis" that incorporates both cause and effect as might be defined by infectious agents and resultant lesions (key determinant causes).

\section{Digital Image Capture}

Digital images were taken during each necropsy to provide a general overview of the carcass, to document specific pathological changes, and to provide data vali-

dation for reported necropsy findings. Digital imaging has been used as part of the collection of necropsy information from feedlots. In feedlot animals, 4 to 8 views should be adequate for establishing a diagnosis, using a standard necropsy technique and comprehensive written protocols (Wildman et al., 2000). In the current study, 3 standard images were taken: the unopened carcass lying on its left side, the opened abdominal cavity, and the opened thoracic cavity. Other images were discretionary and represented notable findings depicting abnormalities documented on the postmortem data sheet. For instance, if the only notable pathological alteration was gross evidence of hemorrhagic bowel syndrome, then an additional digital image was taken of the affected intestinal section.

\section{Conceptually Modeling Postmortem Findings}

Results from the postmortem evaluation were conceptually modeled as described by Lescourret et al. (1993). Such a model provides analysis at a conceptual level in that it is free of the constraints of database 
Table 1. Descriptive statistics and chi-squared analysis of annual dairy cow deaths with no necropsy examination versus deaths with a necropsy examination on 3 dairies separately and in combination by parity and days postpartum ${ }^{1}$

\begin{tabular}{|c|c|c|c|c|c|c|c|c|c|c|c|c|}
\hline \multirow{2}{*}{ Item } & \multicolumn{3}{|c|}{ Dairy A } & \multicolumn{3}{|c|}{ Dairy B } & \multicolumn{3}{|c|}{ Dairy C } & \multicolumn{3}{|c|}{ Combined dairies } \\
\hline & \multicolumn{2}{|c|}{ Necropsy, n } & $\begin{array}{c}\chi^{2} \\
P \text {-value }\end{array}$ & \multicolumn{2}{|c|}{ Necropsy, n } & $\chi^{2} P$-value & \multicolumn{2}{|c|}{ Necropsy, n } & $\chi^{2} P$-value & \multicolumn{2}{|c|}{ Necropsy, n } & $\chi^{2} P$-value \\
\hline Total per dairy & 43 & 68 & \multirow{3}{*}{0.08} & 21 & 39 & \multirow{3}{*}{0.97} & 206 & 67 & \multirow{3}{*}{0.97} & 270 & 174 & \multirow{3}{*}{0.51} \\
\hline Parity & & & & & & & & & & & & \\
\hline 1 & 19 & 17 & & 5 & 9 & & 58 & 18 & & 82 & 44 & \\
\hline \multicolumn{3}{|l|}{ Days postpartum } & \multirow[t]{5}{*}{0.36} & & & \multirow[t]{5}{*}{1.00} & & & 0.88 & & & \multirow{5}{*}{0.46} \\
\hline$\leq 15$ & 8 & 18 & & 6 & 11 & & 43 & 16 & & 57 & 45 & \\
\hline $16-30$ & 5 & 5 & & 4 & 7 & & 23 & 9 & & 32 & 21 & \\
\hline $31-60$ & 5 & 3 & & 3 & 5 & & 28 & 8 & & 36 & 16 & \\
\hline$>60$ & 25 & 42 & & 8 & 16 & & 112 & 34 & & 145 & 92 & \\
\hline
\end{tabular}

${ }^{1}$ Due to multiple comparisons per category, a $P$-value of $\leq 0.01$ was used to establish significant differences.

management system implementation; a single model can be translated into different data structures. A representation of this conceptual data modeling is shown in Figure 2 and was based on the entity-relationship approach, with entities describing real objects (cow, dairy) and relationships providing connections between such entities (i.e., cows belong to a dairy). In this model, the entity cow was defined by several concrete characteristics or attributes. Similarly, the entity dairy consisted of several potentially influential dynamic attributes. The relationship between the dairy and the cow also contained an attribute establishing the period of time that the cow was on the farm. However, the relationship between the dairy and the cow primarily served as a conduit for incorporating influential operational characteristics into the postmortem evaluation. Categories were then formulated on this complete representation of the individual animal and dairy attributes such that a relationship formed between each death and a categorical descriptor for that death. This relationship between the cow and a death category was defined by an attribute based on the specificity of the cause of death. This relational attribute reflected whether a particular mortality was most effectively categorized via a proximate cause such as a specific disease or a key determinant cause founded on more general temporal or managerial influences. Themes were then applied to like categories based on a relational attribute describing the type of death in terms of problems related to clinical disease, disease recognition or treatment, trauma, nutrition, or the stage of lactation.

\section{Comparisons Between Dairies}

For comparison, each dairy's annual mortality and sold-to-slaughter percentages were calculated using the total number of dead and sold cows, respectively, over the 12-mo period extending from November 1, 2006, to October 31, 2007, divided by the number of dairy cows present on the operation at the end of that period. For each dairy and for the combined dairies, the distributions of total annual necropsied deaths by parity and by days postpartum were compared with the distributions of nonnecropsied deaths using a chi-squared test (PROC FREQ, SAS, version 9.1, SAS Inst. Inc., Cary, NC). In addition, the distribution of deaths by parity, days postpartum, lactation status, pregnancy status, type of death (killed versus unassisted), and recumbency $\geq 24$ $\mathrm{h}$ before death were compared among dairies using chisquared testing. Similarly, the distribution of specific and combined health events and treatments that were recorded per necropsied animal were compared among dairies, as were the distribution of anatomic systems with pathologic necropsy findings, the primary anatomic pathology associated with death, and the distribution of necropsied animals placed within specific categorical themes. For all comparisons in which more than $20 \%$ of contingency table cell counts were less than 5 , SAS PROC FREQ computed Fisher's exact test using the network algorithm of Mehta and Patel (1983). For evaluations of a single null hypothesis, $P \leq 0.05$ was considered statistically significant. For multiple comparisons, the significance level was adjusted by dividing the desired significance level $(0.05)$ by the number of null hypotheses evaluated.

\section{RESULTS}

\section{Postmortem Comparisons of Life-History Features}

During the study, 174 postmortem evaluations were performed. Dairy C's involvement was restricted to 4 mo because of the departure of the in-house veterinar- 
ian. Of the 174 postmortems, 68 were performed on dairy $\mathrm{A}, 39$ on dairy $\mathrm{B}$, and 67 on dairy $\mathrm{C}$ (Table 1 ). Of those 39 postmortems on dairy B, 7 were from Jerseys as opposed to Holsteins, a similar distribution to the breed distribution within the herd and comprising a relatively small sample from which to make meaningful comparisons based on breeds. Total deaths on dairies $\mathrm{A}, \mathrm{B}$, and $\mathrm{C}$ over a 12 -mo period extending from November 1, 2006, to October 31, 2007, were 111, 60, and 273 , respectively. Herd inventories for the 3 dairies were 1,529 (A), 777 (B), and 2,255 (C) on October 31, 2007. Annual mortality percentages for dairies A, B, and C were $7.3,7.7$, and $12.1 \%$ and the annual percentages of cattle sold to slaughter were $31.5,17.4$, and $30.4 \%$, respectively. The consequent percentages of overall removals that resulted from death loss rather than other, more economically favorable culling decisions equated to $18.8,30.8$, and $28.5 \%$ for dairies $\mathrm{A}, \mathrm{B}$, and $\mathrm{C}$, respectively. The distribution of necropsied deaths by parity did not differ from the distribution of annual nonnecropsied deaths by parity (Table 1 ). Nor was the distribution of necropsied cows by days postpartum different from the distribution of annual deaths by days postpartum (Table 1). The distribution of necropsied cows by parity, days postpartum, pregnancy status, and recumbency status before death were not dependent on the dairy (Table 2). However, the distributions of necropsied cows by lactation status and type of death were dependent on the dairy (Table 2).

\section{Postmortem Comparisons of Health Events, Treatments, and Necropsy Findings}

Relevant health events and treatments were recorded for each of the 174 deaths. The distribution of deaths by specific health events and treatments showed some dependency on the dairy (Table 3). Occurrences of milk fever and lameness varied according to dairy. When health events were categorized by the number of occurrences per animal $(0,1,2,>2)$, there was no dependence on the dairy. Of the treatments given, only the use of anti-inflammatories, intravenous fluids and electrolytes, and vitamins varied according to dairy. When treatments were categorized by the number of occurrences per animal $(0,1,2,>2)$, the categories representing 2 and $>2$ varied with dairy. The distribution of deaths by specific anatomic lesion diagnoses and the anatomic system with the primary pathology associated with death demonstrated some variation among dairies (Table 3). Pathological changes associated with the abdomen or peritoneum, abomasum, heart, liver, lungs, oral cavity, and trachea varied with dairy. The

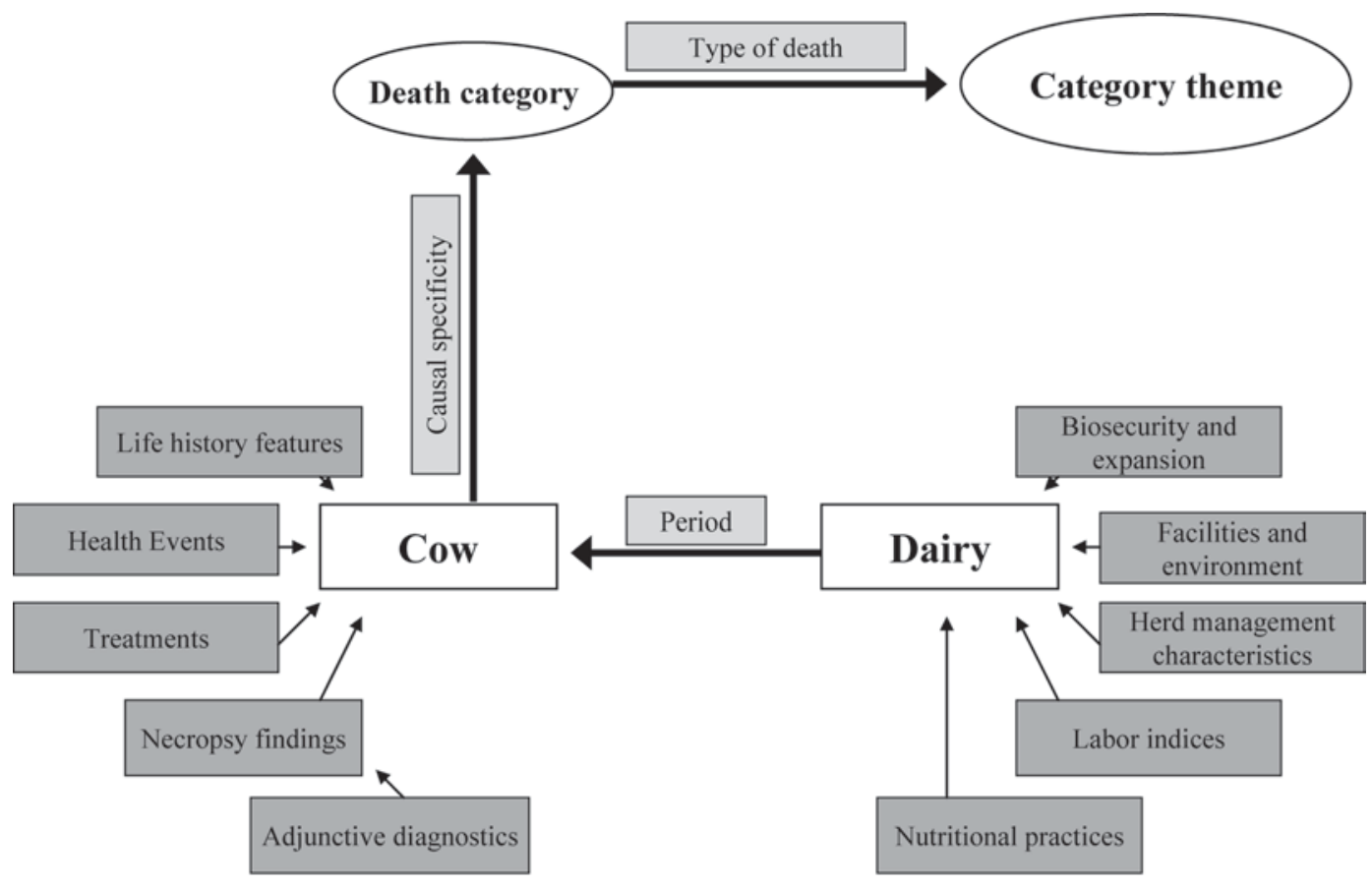

Concrete data

Dynamic data

Figure 2. Conceptual data model used to organize necropsy-based postmortem information into a categorical scheme developed for monitoring dairy cow mortality. 
Table 2. Descriptive statistics and chi-squared analysis from 174 deaths on 3 dairies by parity, days postpartum, lactation status, pregnancy status, type of death, and recumbency status before death ${ }^{1}$

\begin{tabular}{|c|c|c|c|c|c|c|c|c|c|}
\hline \multirow[b]{2}{*}{ Item } & \multicolumn{2}{|c|}{ Dairy A } & \multicolumn{2}{|c|}{ Dairy B } & \multicolumn{2}{|c|}{ Dairy C } & \multirow[b]{2}{*}{$\chi^{2} P$-value } & \multicolumn{2}{|c|}{ Combined dairies } \\
\hline & $\mathrm{n}$ & $\%$ & $\mathrm{n}$ & $\%$ & $\mathrm{n}$ & $\%$ & & $\mathrm{n}$ & $\%$ \\
\hline 1 & 17 & 25 & 9 & 23 & 18 & 27 & & 44 & 25 \\
\hline $2-4$ & 43 & 63 & 18 & 46 & 37 & 55 & & 98 & 56 \\
\hline$\geq 5$ & 8 & 12 & 12 & 31 & 12 & 18 & & 32 & 18 \\
\hline Days postpartum & & & & & & & 0.27 & & \\
\hline $31-60$ & 3 & 4 & 5 & 13 & 8 & 12 & & 16 & 9 \\
\hline$>60$ & 42 & 62 & 16 & 41 & 34 & 51 & & 92 & 53 \\
\hline Lactation status & & & & & & & 0.03 & & \\
\hline Lactating & 66 & 97 & 38 & 97 & 58 & 87 & & 162 & 93 \\
\hline Dry & 2 & 3 & 1 & 3 & 9 & 13 & & 12 & 7 \\
\hline Pregnancy status & & & & & & & 0.21 & & \\
\hline Pregnant & 15 & 22 & 4 & 10 & 16 & 24 & & 35 & 20 \\
\hline Yes & 18 & 26 & 16 & 41 & 24 & 36 & & 58 & 33 \\
\hline No & 50 & 74 & 23 & 59 & 43 & 64 & & 116 & 67 \\
\hline
\end{tabular}

${ }^{1} \mathrm{~A} P$-value of $\leq 0.05$ was used to establish significant differences.

anatomic system listed as the primary pathology associated with death differed among dairies for the small intestine. The number of anatomic systems with pathologic abnormalities per death $(0,1,2,3,4,5,>5)$ was not dependent upon the dairy.

\section{Digital Image Utility}

Digital image documentation of dairy cow necropsies proved to be a complicated endeavor in large part because of the often varied disease processes culminating in a death. As shown in Table 3, over a third of deaths $(36 \%)$ demonstrated 5 or more systems with evidence of pathology. Although not all documented pathologies were integral to the final outcome of death, capturing the relevant affected anatomic systems required a detailed examination with a thorough understanding of bovine physiology. Although many images clearly captured a specific pathology, capturing all significant pathologies within an individual animal and providing sufficient explanation for images that failed to demonstrate pathologic context was difficult. For instance, ruptured vessels were easily enough observed within an image but often required a detailed explanation of the anatomic location and other potential pathologic influences if the cause of death was to be more fully understood. Ultimately, the digital images provided a discussion point when reviewing individual deaths but were not useful as the sole explanation of pertinent lesions. Their utility was limited by the complexities of the necropsy examination and reliant on thorough input from the prosector.

\section{Dairy and Veterinarian Differences}

Although the majority of comparisons between dairies for life-history features, health events, treatments, and necropsy findings were not significantly different, the differences identify difficulties in reliably documenting the complexities associated with dairy postmortems. The differences between dairies likely represented variations in operational management and environment and veterinary perspective. For instance, dairy A likely documented greater levels of oral cavity and tracheal pathologies because a veterinarian on dairy A more closely scrutinized those anatomic systems, not because of a disease complex specific to that dairy. This potential for relativism underscores the importance of establishing an information system that views each death as a whole. For this study, this included the assessment by the participating veterinarians of more dynamic data related to operational management and considered important to the final outcome. Although still subjective, these data provided a more complete picture of the events leading up to the death such that each death could be viewed within the context of the complexity of a cow's life on a dairy.

\section{Conceptual Model}

Relevant attributes related to the dairy and cow entities and the relationship between them were 
Table 3. Descriptive statistics and chi-squared analysis from 174 deaths on 3 dairies by specific health events, health events categorized by number of occurrences per animal, treatments, treatments categorized by number of occurrences per animal, specific anatomic systems with pathologic necropsy findings, the number of anatomic systems with pathologic abnormalities per death, and the anatomic system with the primary pathology associated with death ${ }^{1}$

Health event occurrences per animal

$(P$ significant $\leq 0.01)$

Treatments

$(P$ significant $\leq 0.005)$

Treatment occurrences per animal

$(P$ significant $\leq 0.01)$

Specific anatomic systems with pathologic necropsy findings $(P$ significant $\leq 0.002)$
Number of anatomic systems with pathologic abnormalities per death $(P$ significant $\leq 0.007)$

\begin{tabular}{|c|c|c|c|c|c|c|c|c|c|}
\hline & \multicolumn{2}{|c|}{ Dairy A } & \multicolumn{2}{|c|}{ Dairy B } & \multicolumn{2}{|c|}{ Dairy C } & \multirow[b]{2}{*}{$\chi^{2} P$-value } & \multicolumn{2}{|c|}{$\begin{array}{c}\text { Combined } \\
\text { dairies }\end{array}$} \\
\hline & $\mathrm{n}$ & $\%$ & $\mathrm{n}$ & $\%$ & $\mathrm{n}$ & $\%$ & & $\mathrm{n}$ & $\%$ \\
\hline Clinical mastitis & 14 & 21 & 12 & 31 & 14 & 21 & 0.42 & 40 & 23 \\
\hline Milk fever & 1 & 1 & 9 & 23 & 4 & 6 & $<0.004^{*}$ & 14 & 8 \\
\hline Ketosis & 4 & 6 & 2 & 5 & 3 & 4 & 1.00 & 9 & 5 \\
\hline Lameness & 6 & 9 & 3 & 8 & 24 & 36 & $<0.004^{*}$ & 33 & 19 \\
\hline Respiratory problems & 10 & 15 & 4 & 10 & 8 & 12 & 0.78 & 22 & 13 \\
\hline Diarrhea $>48 \mathrm{~h}$ & 3 & 4 & 5 & 13 & 5 & 7 & 0.28 & 13 & 7 \\
\hline Melena: ulcers/HBS ${ }^{2}$ & 1 & 1 & 2 & 5 & 0 & 0 & 0.24 & 3 & 2 \\
\hline Abortion & 3 & 4 & 2 & 5 & 1 & 1 & 0.65 & 6 & 3 \\
\hline Retained placenta & 2 & 3 & 5 & 13 & 5 & 7 & 0.15 & 12 & 7 \\
\hline Dystocia & 12 & 18 & 8 & 21 & 13 & 19 & 0.93 & 33 & 19 \\
\hline Metritis & 9 & 13 & 7 & 18 & 12 & 18 & 0.71 & 28 & 16 \\
\hline Displaced abomasum & 8 & 12 & 1 & 3 & 16 & 24 & 0.01 & 25 & 14 \\
\hline Neurological problems & 1 & 1 & 1 & 3 & 1 & 1 & 1.00 & 3 & 2 \\
\hline 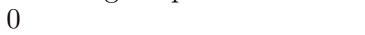 & 16 & 24 & 10 & 26 & 8 & 12 & 0.13 & 34 & 20 \\
\hline 1 & 35 & 51 & 13 & 33 & 27 & 40 & 0.16 & 75 & 43 \\
\hline 2 & 12 & 18 & 7 & 18 & 21 & 31 & 0.12 & 40 & 23 \\
\hline$>2$ & 5 & 7 & 9 & 23 & 11 & 16 & 0.07 & 25 & 14 \\
\hline No treatments & 12 & 18 & 2 & 5 & 6 & 9 & 0.10 & 20 & 11 \\
\hline Antibiotics (IM, IV, SC) ${ }^{3}$ & 28 & 41 & 25 & 64 & 38 & 57 & 0.05 & 91 & 52 \\
\hline Intramammary antibiotics & 5 & 7 & 4 & 10 & 8 & 12 & 0.66 & 17 & 10 \\
\hline Intrauterine antibiotic/flush & 1 & 1 & 0 & 0 & 2 & 3 & 0.62 & 3 & 2 \\
\hline Anti-inflammatories & 25 & 37 & 32 & 82 & 47 & 70 & $<0.005^{*}$ & 104 & 60 \\
\hline IV fluids/electrolytes & 16 & 24 & 20 & 51 & 38 & 57 & $<0.005^{*}$ & 74 & 43 \\
\hline Oral fluids/electrolytes & 26 & 38 & 19 & 49 & 37 & 55 & 0.14 & 82 & 47 \\
\hline Vitamins & 1 & 1 & 5 & 13 & 30 & 45 & $<0.005^{*}$ & 36 & 21 \\
\hline Hoof block/trim & 2 & 3 & 1 & 3 & 6 & 9 & 0.28 & 9 & 5 \\
\hline Surgical intervention & 7 & 10 & 2 & 5 & 16 & 24 & 0.01 & 25 & 14 \\
\hline 0 & 18 & 26 & 3 & 8 & 9 & 13 & 0.03 & 30 & 17 \\
\hline 1 & 12 & 18 & 7 & 18 & 13 & 19 & 0.96 & 32 & 18 \\
\hline 2 & 22 & 32 & 9 & 23 & 6 & 9 & $<0.01^{*}$ & 37 & 21 \\
\hline$>2$ & 16 & 24 & 20 & 51 & 39 & 58 & $<0.01^{*}$ & 75 & 43 \\
\hline No abnormalities & 2 & 3 & 1 & 3 & 0 & 0 & 0.44 & 3 & 2 \\
\hline Abdomen/peritoneum & 33 & 49 & 22 & 56 & 13 & 19 & $<0.002 *$ & 68 & 39 \\
\hline Abomasum & 22 & 32 & 5 & 13 & 43 & 64 & $<0.002^{*}$ & 70 & 40 \\
\hline Bladder & 0 & 0 & 1 & 3 & 0 & 0 & 0.22 & 1 & 1 \\
\hline Brain & 0 & 0 & 0 & 0 & 1 & 1 & 0.61 & 1 & 1 \\
\hline Esophagus & 3 & 4 & 0 & 0 & 1 & 1 & 0.45 & 4 & 2 \\
\hline Eyes & 4 & 6 & 4 & 10 & 2 & 3 & 0.28 & 10 & 6 \\
\hline Heart & 29 & 43 & 8 & 21 & 38 & 57 & $<0.002^{*}$ & 75 & 43 \\
\hline Kidneys & 6 & 9 & 1 & 3 & 4 & 6 & 0.53 & 11 & 6 \\
\hline Large intestine/cecum & 5 & 7 & 3 & 8 & 3 & 4 & 0.78 & 11 & 6 \\
\hline Liver & 16 & 24 & 18 & 46 & 38 & 57 & $<0.002^{*}$ & 72 & 41 \\
\hline Lungs & 37 & 54 & 10 & 26 & 41 & 61 & $<0.002^{*}$ & 88 & 51 \\
\hline Musculoskeletal & 15 & 22 & 11 & 28 & 23 & 34 & 0.28 & 49 & 28 \\
\hline Oral cavity & 8 & 12 & 0 & 0 & 0 & 0 & $<0.002^{*}$ & 8 & 5 \\
\hline Rumen, reticulum, omasum & 12 & 18 & 1 & 3 & 6 & 9 & 0.04 & 19 & 11 \\
\hline Small intestine & 20 & 29 & 15 & 38 & 12 & 18 & 0.06 & 47 & 27 \\
\hline Spleen & 1 & 1 & 0 & 0 & 1 & 1 & 1.00 & 2 & 1 \\
\hline Systemic & 25 & 37 & 16 & 41 & 24 & 36 & 0.86 & 65 & 37 \\
\hline Trachea & 12 & 18 & 0 & 0 & 2 & 3 & $<0.002^{*}$ & 14 & 8 \\
\hline Udder & 18 & 26 & 10 & 26 & 12 & 18 & 0.45 & 40 & 23 \\
\hline Uterus & 15 & 22 & 12 & 31 & 21 & 31 & 0.43 & 48 & 28 \\
\hline 0 & 0 & 0 & 1 & 3 & 0 & 0 & 0.22 & 1 & 1 \\
\hline 1 & 8 & 12 & 4 & 10 & 3 & 4 & 0.35 & 15 & 9 \\
\hline 2 & 11 & 16 & 6 & 15 & 9 & 13 & 0.90 & 26 & 15 \\
\hline 3 & 12 & 18 & 10 & 26 & 11 & 16 & 0.47 & 33 & 19 \\
\hline 4 & 12 & 18 & 6 & 15 & 18 & 27 & 0.27 & 36 & 21 \\
\hline 5 & 6 & 9 & 9 & 23 & 10 & 15 & 0.13 & 25 & 14 \\
\hline$>5$ & 19 & 28 & 3 & 8 & 16 & 24 & 0.04 & 38 & 22 \\
\hline
\end{tabular}


Table 3 (Continued). Descriptive statistics and chi-squared analysis from 174 deaths on 3 dairies by specific health events, health events categorized by number of occurrences per animal, treatments, treatments categorized by number of occurrences per animal, specific anatomic systems with pathologic necropsy findings, the number of anatomic systems with pathologic abnormalities per death, and the anatomic system with the primary pathology associated with death ${ }^{1}$

\begin{tabular}{|c|c|c|c|c|c|c|c|c|c|c|}
\hline \multirow[b]{2}{*}{ Item } & & \multicolumn{2}{|c|}{ Dairy A } & \multicolumn{2}{|c|}{ Dairy B } & \multicolumn{2}{|c|}{ Dairy C } & \multirow[b]{2}{*}{$\chi^{2} P$-value } & \multicolumn{2}{|c|}{$\begin{array}{l}\text { Combined } \\
\text { dairies }\end{array}$} \\
\hline & & $\mathrm{n}$ & $\%$ & $\mathrm{n}$ & $\%$ & $\mathrm{n}$ & $\%$ & & $\mathrm{n}$ & $\%$ \\
\hline the primary pathology & Abomasum & 7 & 10 & 2 & 5 & 5 & 7 & 0.62 & 14 & 8 \\
\hline associated with death & Eyes & 0 & 0 & 1 & 3 & 0 & 0 & 0.22 & 1 & 1 \\
\hline \multirow[t]{8}{*}{$(P$ significant $\leq 0.004)$} & Heart & 4 & 6 & 1 & 3 & 4 & 6 & 0.82 & 9 & 5 \\
\hline & Musculoskeletal & 8 & 12 & 5 & 13 & 15 & 22 & 0.20 & 28 & 16 \\
\hline & Rumen, reticulum, omasum & 5 & 7 & 0 & 0 & 1 & 1 & 0.15 & 6 & 3 \\
\hline & Small intestine & 3 & 4 & 11 & 28 & 7 & 10 & $<0.004^{*}$ & 21 & 12 \\
\hline & Systemic & 11 & 16 & 2 & 5 & 3 & 4 & 0.06 & 16 & 9 \\
\hline & Udder & 3 & 4 & 0 & 0 & 3 & 4 & 0.51 & 6 & 3 \\
\hline & Uterus & 3 & 4 & 7 & 18 & 4 & 6 & 0.05 & 14 & 8 \\
\hline & Undetermined & 2 & 3 & 1 & 3 & 0 & 0 & 0.44 & 3 & 2 \\
\hline
\end{tabular}

\footnotetext{
${ }^{1}$ Assessment of relevant health and treatment events was carried out by the attending veterinarian and was focused on capturing information believed to have potentially influenced the final outcome of death. Due to multiple comparisons, the significance level of $P$-values was adjusted by dividing the desired significance level $(0.05)$ by the number of null hypotheses evaluated per category.

${ }^{2} \mathrm{HBS}=$ hemorrhagic bowel syndrome.

${ }^{3} \mathrm{IM}=$ intramuscular; $\mathrm{IV}=$ intravenous; $\mathrm{SC}=$ subcutaneous.

* Significant $P$-value based on multiple comparisons.
}

documented and applied to the conceptual model demonstrated in Figure 2. Each death was viewed in the context of the web of factors influencing the dairy and the cow. Categories were formulated in an effort to create a monitoring system describing mortality in terms of functional characteristics potentially amenable to performance evaluation, management oversight, and research.

As an example, a 3-yr-old, primiparous, 21-dpostpartum Holstein was killed on January 11, 2007 (life-history features). Her death followed an initially transient period of recumbency several days postpartum that was accompanied by a rapid loss in condition and eventuated in permanent recumbency for a 2-d period before euthanasia (health events). She had been treated with oral and intravenous fluids, antiinflammatories, and injectable vitamins and had been floated in a water tank to mitigate side effects from recumbency (treatments). The postmortem examination demonstrated evidence of abomasal hyperemia, acute bronchopneumonia, metritis, and a moderate hepatic lipidosis (necropsy findings). A pertinent dairy attribute included the purchase of replacements (biosecurity and expansion), which included this heifer from a dairy that failed to breed her in a timely fashion, resulting in overconditioning and a late age at first calving. An additional and variable dairy attribute included a prolonged period of inclement weather at the time of this animal's demise (facilities and environment). The various concrete and dynamic attributes influencing this animal's death suggested that the causal specificity attribute within the relationship between the entities cow and death category was less a function of a specific proximate cause (i.e., a particular disease process) and more the result of key determinant issues related to the period of early lactation (i.e., multifactorial negative influences). The combination of an older, over-conditioned heifer at the time of calving; stress associated with an environmental change and inclement weather; and consequent immunologic, hepatic, and pulmonic pathologies terminated in permanent recumbency requiring euthanasia. Therefore, this particular death was included within a category incorporating the multifactorial reasons for transition failure. The final relationship between this death category and its overarching theme was dictated by the type of death attribute's relationship to the stage of lactation, in this case specifically targeting transition cow or negative energy balance issues.

\section{Specific Disease Processes with Variable Etiologies}

In total, 21 death categories with 7 category themes were formulated based on the model guidelines. There were several disease processes that stood alone as proximate causes of death but might or might not have had manageable etiologies originating with other underlying determinants. Thematically, this set of categories encompassed specific disease processes with variable etiologies (Table 4). The explicit pathophysiologic attributes required specific categories, yet the majority 
Table 4. Descriptive statistics, chi-squared analysis, and mortality categories and themes describing 174 deaths on 3 dairies ${ }^{1}$

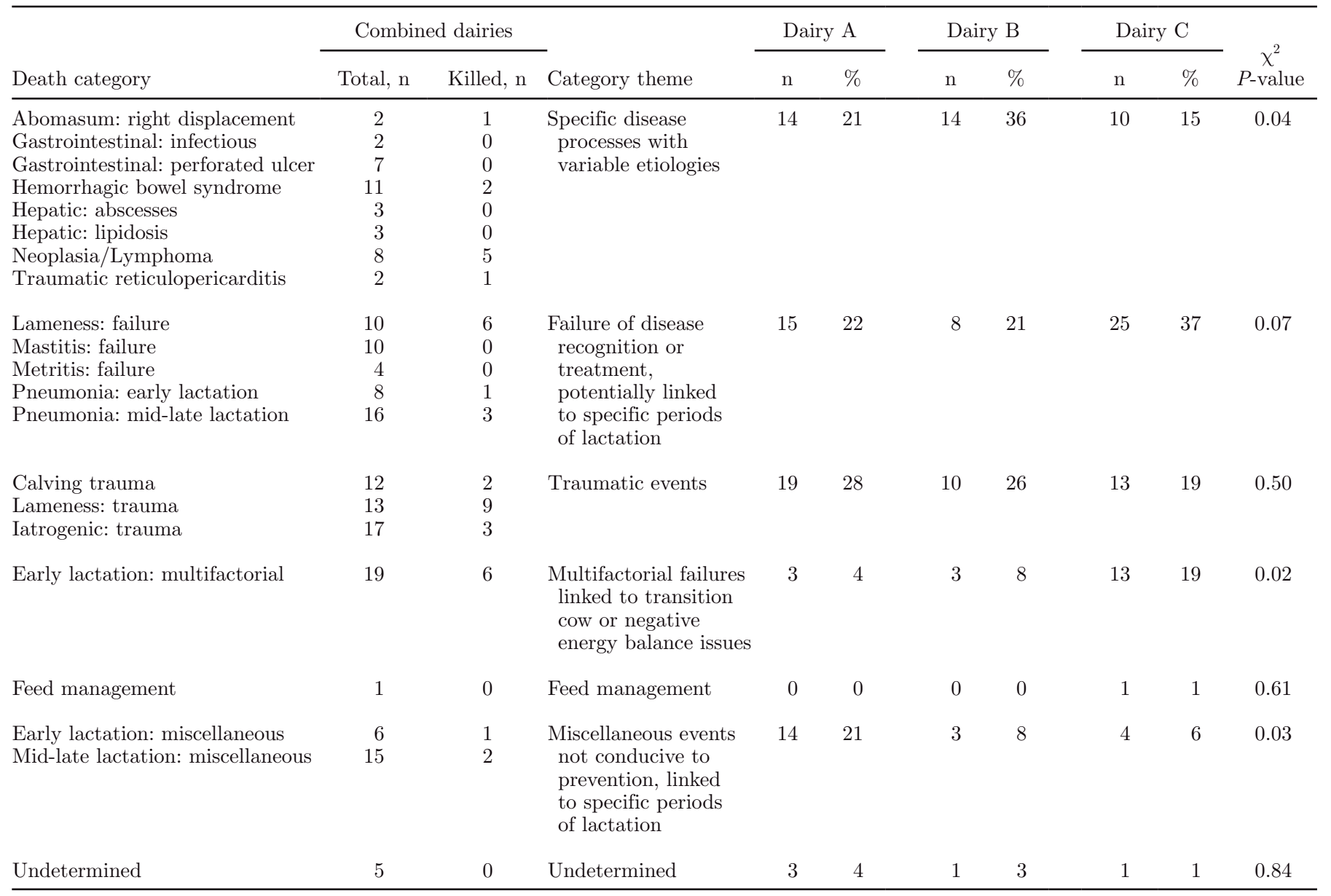

${ }^{1}$ Categories that refer to early and mid-late lactation periods encompass $\leq 60$ DIM and $>60$ DIM, respectively. A $P$-value of $\leq 0.05$ was used to establish significant differences.

of these pathologies were undoubtedly manifestations of multifactorial problems. However, under the circumstances, they provided a specific action or analytic point when clearly evident and clearly severe. Rather than bury specifics in generalities (i.e., the multifactorial and miscellaneous categories to follow), these disease entities were left as a stand-alone category if they captured the essence of the underlying etiology or if there was evidence that they might be amenable to management alternatives.

\section{Failure of Disease Recognition or Treatment}

Some initiating factors that eventuated in death were diseases for which there was a failure of recognition or treatment (Table 4). These problems could all be deconstructed into the periods of lactation in which they occurred, but for the purposes of this study the subcategorization appeared primarily relevant to pneumonia. The categories included failures in lameness (primarily referring to chronic digital infections - not injury or trauma), mastitis, metritis, and pneumonia. The cases of pneumonia were further broken into lactational periods (early lactation: $\leq 60 \mathrm{DIM}$, mid/late lactation: $>60$ DIM), the purpose of which was to provide a delineation of the failed outcome not only in terms of the disease but with respect to relevant periods with inherent health challenges from which the disease problem arose. For instance, pneumonia during the early transitional period might be influenced by specific factors related to close-up maternity management, whereas late-lactational pneumonia might result from a very different set of underlying factors. Whereas the temporal aspect of this categorization provided the context for the pathophysiology, the disease remained the target for directing management alternatives. This necessitated categories dictated by the failed disease intervention rather than the period during which the disease occurred. 


\section{Traumatic Events}

The majority of deaths resulting from traumatic events were a consequence of trauma associated with parturition or injuries resulting in lameness (Table 4). This categorical theme also included the category iatrogenic: trauma. Although this covered a fairly wide arena (aspiration pneumonia, injection site abscesses, drug reactions, and surgical complications), the significance of this category was in its documentation of those deaths that occurred not because of the initiating disease process but because of the attempt to treat that process. If those failures were ignored, it would be too easy to blame a loss on an initial episode of pneumonia or a displaced abomasum rather than dealing with the underlying issue of worker training or surgical competence. As might be expected, this categorical theme represented more euthanasias than did any of the other themes $(14 / 42)$, with the death category lameness: trauma accounting for more euthanasias (9) than any other specific category (Table 4).

\section{Multifactorial Failures Linked to Transition or Negative Energy Balance Issues}

The categorical theme with the most thorough incorporation of concrete and dynamic attributes encompassed multifactorial failures linked to transition cow or negative energy balance issues (Table 4). This subset of deaths represented early-lactation failures following any combination of health events such as ketosis, diarrhea, milk fever, respiratory disease, retained placenta, metritis, poor body condition, and electrolyte imbalancesnot one of which specifically led to any particular death but all of which contributed. These deaths could not be ascribed to any one cause because of the multiplicity of pathologies; rather, they represented a failure in the preparation for and adjustment to the early stages of lactation. As such, specific dynamic attributes of the dairy during these peripartum stages and the consequent relationship with the cow were critically influential in the final outcome of death.

\section{Feed Management, Miscellaneous Events, Undetermined Causes}

Three additional themes completed the categorization of deaths from this study (Table 4). These included an error in feed management resulting from improper mixing and delivery of barley within a TMR and miscellaneous findings that were not clearly preventable or treatable but could be broken into early ( $\leq 60$ DIM) and late ( $>60$ DIM) periods of lactation. The miscellaneous early-lactational losses were not obviously attributed to transition or negative energy balance issues and were not easily defined in terms of the pathologic sequence but tended to be the result of infectious processes (e.g., flank myositis, peritonitis, or pericarditis) with unknown initiating causes. On the other hand, the latelactational findings were typically the result of random and unmanageable events including a ruptured abdominal abscess of unknown origin, gastric vein rupture, and late-gestational uterine rupture. The final theme included postmortem evaluations that demonstrated no proximate or key determinant causes of death such that the reasons for the deaths were left undetermined.

\section{DISCUSSION}

\section{The Problem of Dairy Cow Mortality}

It is time to rethink how the dairy industry approaches the issue of excessive mortality. Even in the face of economic and ethical concerns, there is little literature specific to the subject and evidence suggests a limited understanding of the reasons why cows die (Thomsen and Houe, 2006; McConnel et al., 2009). A tacit acceptance of the problem pervades the industry; to paraphrase, bad has become normal (Grandin and Johnson, 2005). Preceding events and dairy dynamics that influence poor outcomes must be simultaneously addressed to tackle this problem (McConnel et al., 2009), yet systems are typically not in place on dairies to accurately track and effectively analyze mortality data. Without a thorough understanding of the cause and effect underlying individual deaths, and a means for monitoring those deaths within a population, accountability cannot be established.

\section{Detailing the Who, What, When, Where, How, and Why of Dairy Cow Deaths}

Historically, the best documented aspect of a cow's death has been its unique life-history features such as identification, lactation number, and DIM. This has typically provided an accurate account of who died. What caused the death is often only partially documented and typically without the benefit of a postmortem examination. However, even using a necropsy to establish the proximate cause of death might not provide adequate insight into the key determinant or underlying causes that eventuated in a death. Moreover, categorizing necropsy findings in a meaningful way is difficult, and current on-farm record systems are not configured to efficiently or effectively capture such information (McConnel et al., 2009). Typically, the when and the where of a cow's death have effectively been left out of the monitoring equation and can be difficult to derive from a database. Although information related to specific temporal events such as calving are recorded, dynamic 
data related to a cow's time on a dairy, pen moves and crowding, environmental exposure, and other attributes establishing the cow's interaction with the dairy might be missing and are often poorly associated with the documented reason for a cow's death. This ultimately results in a profound lack of understanding of how and why a cow truly died.

\section{Creating Accuracy and Consistency in Record Keeping}

Studies have explored systems for recording specific clinical diseases associated with dairy cows (Kaneene and Hurd, 1990; Kelton et al., 1998; Osteras et al., 2007). Difficulties arise from inconsistent standards for disease definitions and data presentation. Such data are necessary if disease is to be described, compared, and investigated on national and regional levels in an effort to efficiently modify the management practices that promote cattle health (Kelton et al., 1998). This need for consistent standards and more in-depth characterization of data has been explored relative to removals as a whole (Fetrow et al., 2006). Further delineation is required if dairy cow mortality is to be specifically and effectively addressed. Current methods for monitoring dairy mortality are variable, inconclusive, and often founded on assumptions (McConnel et al., 2009). The complexity inherent in dairy operations necessitates the incorporation of database models that rationalize the system. Through modeling, it is possible to incorporate all attributes of dairy (herd-level) and cow (animallevel) entities into a rational explanation for a death. This accounting of mortality expands the equation to focus not only on how and why cows die but what can be done about it through the establishment of critical control points that can be targeted to mitigate losses.

The current study was derived from principles established in previous investigations (Thomsen and Houe, 2006; McConnel et al., 2008; McConnel et al., 2009). The development of nomenclature for why cows die has originated within pathophysiologic or anatomic descriptors, summing up individual occurrences of death based on the final outcome. Yet even that final outcome has historically been poorly defined and without the aid of a thorough postmortem examination. In addition, links between a death and instigating attributes such as those associated with dairy management or a cow's health history have been overlooked or left out of records meant to describe and monitor mortality. Attention must be focused on those cows most at risk for disease and death, health events must be tracked and recorded, and proximate causes of death based on necropsy findings must be combined with an understanding of those facets of management that influence poor outcomes (McConnel et al., 2009). The continuum of events and failures that eventuate in a death must be appropriately modeled if a database is to be designed that can accurately and thoroughly monitor mortality on dairies. Ultimately, it is the process leading to a death that captures the essence of why a cow died and provides the necessary insight into how best to prevent future similar occurrences.

Although the postmortem evaluations in this study provided a representative sample of the participating dairies' dead cows (Table 1), the inclusion of a limited number of dairies within one region was not expected to provide a generalizable assessment of causes of mortality industry wide. Nor were the categorical descriptors derived from those deaths meant to provide a definitive nomenclature on which to base future monitoring systems. Rather, the model that was developed provides a foundation for pursuing database schemes that can more effectively monitor dairy cow mortality. The concrete and dynamic data (Figure 2) underlying a postmortem evaluation provides the structural integrity necessary for framing a cow's death; however, without database development that can capture these components for future evaluation there is little directive for guiding management alternatives.

\section{CONCLUSIONS}

The aphorism that "those who cannot remember the past are condemned to repeat it" (Santayana, 1917) appears particularly poignant with regard to the problem of dairy cow mortality. Until appropriate monitoring systems are developed, there is little hope for establishing the systemic accountability necessary to direct change, and there is every indication that this challenge will continue to afflict the industry as a whole. However, what might prove a challenging endeavor at the industry level certainly does not preclude individual operations from addressing this issue using knowledge at hand to formulate a best path forward. It is clear from this study and previous work in the area of dairy cow mortality that there are numerous underlying causes of cow deaths and an even greater number of ways to describe those deaths (Thomsen and Houe, 2006; McConnel et al., 2009). Yet even in the face of the various derivations for tracking mortality, there are a few sound suggestions derived from these studies that might prove useful for combating this problem.

Formulate a strategy for performing thorough postmortem evaluations. Target those deaths that lie outside of obvious instances (accidents, locomotor disorders, euthanasias) so that the information gained warrants the cost and effort required to perform a necropsy. Incorporate farm employees into the process as a teaching tool to stimulate interest in the problem and as a means 
of demonstrating poor outcomes from potentially poor decisions. Use hard-copy records such as those demonstrated in the current study (Figure 1) to capture as much detail as possible related to an individual death. Take the time to record dynamic aspects influencing a death that might not be available in standard record systems. Take digital photos to provide clarification for future questions that might arise regarding certain deaths. Although mortality levels are generally greater than desired, the numbers are typically not so great that data sheets and photo documentation cannot be stored for future analysis.

Standardize health event nomenclature in simple and consistent terms that will provide useful background information not only for the analysis of deaths but other health-related questions as well. Consider developing a coding system for deaths based on categories such as those described in this study and suitably tailored to an individual farm's challenges. An appropriate categorization scheme can partition overly specific details or apparently unmanageable generalities into functional themes. Record these codes on on-farm computer systems and organize hard copy necropsy sheets and digital photography accordingly for future reference and analysis. Taking such measures can provide direction for addressing problems as they arise. Perhaps as importantly, unpublished data and anecdotal evidence suggest that making progress toward resolving this issue might simply require acknowledging its importance. The act of recognizing rising mortality as a problem might, in fact, be the most fundamental step toward controlling its progression.

\section{ACKNOWLEDGMENTS}

This research was funded, in part, by the Colorado State University Agricultural Experiment Station and the Colorado State University Animal Population Health Institute. The authors are sincerely indebted to the owners and personnel of the 3 dairies and to the participating veterinarians for their invaluable collaboration during the study.

\section{REFERENCES}

Bar, D., Y. T. Grohn, G. Bennett, R. N. Gonzalez, J. A. Hertl, H. F. Schulte, L. W. Tauer, F. L. Welcome, and Y. H. Schukken. 2008. Effects of repeated episodes of generic clinical mastitis on mortality and culling in dairy cows. J. Dairy Sci. 91:2196-2204.

Dechow, C. D., and R. C. Goodling. 2008. Mortality, culling by sixty days in milk, and production profiles in high- and low-survival Pennsylvania herds. J. Dairy Sci. 91:4630-4639.

Fetrow, J., K. V. Nordlund, and H. D. Norman. 2006. Invited review: Culling: nomenclature, definitions, and recommendations. J. Dairy Sci. 89:1896-1905.

Grandin, T., and C. Johnson. 2005. Animals in Translation: Using the Mysteries of Autism to Decode Animal Behavior. Scribner, New York, NY.
Hare, E., H. D. Norman, and J. R. Wright. 2006. Survival rates and productive herd life of dairy cattle in the United States. J. Dairy Sci. 89:3713-3720.

Kaneene, J. B., and H. S. Hurd. 1990. The National Animal Health Monitoring System in Michigan. I. Design, data and frequencies of selected dairy cattle diseases. Prev. Vet. Med. 8:103-114.

Kelton, D. F. 2006. Epidemiology: A foundation for dairy production medicine. Vet. Clin. North Am. Food Anim. Pract. 22:21-33.

Kelton, D. F., K. D. Lissemore, and R. E. Martin. 1998. Recommendations for recording and calculating the incidence of selected clinical diseases of dairy cattle. J. Dairy Sci. 81:2502-2509.

Lescourret, F., M. Genest, J. Barnouin, M. Chassagne, and B. Faye. 1993. Data modeling for database design in production and health monitoring systems for dairy herds. J. Dairy Sci. 76:1053-1062.

Loneragan, G. H., D. A. Dargatz, P. S. Morley, and M. A. Smith. 2001. Trends in mortality ratios among cattle in US feedlots. J. Am. Vet. Med. Assoc. 219:1122-1127.

Mason, G. L., and D. J. Madden. 2007. Performing the field necropsy examination. Vet. Clin. North Am. Food Anim. Pract. 23:503526.

McConnel, C. S., F. B. Garry, J. E. Lombard, J. A. Kidd, A. E. Hill, and D. H. Gould. 2009. A necropsy-based descriptive study of dairy cow deaths on a Colorado dairy. J. Dairy Sci. 92:1954-1962.

McConnel, C. S., J. E. Lombard, B. A. Wagner, and F. B. Garry. 2008. Evaluation of factors associated with increased dairy cow mortality on United States dairy operations. J. Dairy Sci. 91:1423-1432.

Mehta, C. R., and N. R. Patel. 1983. A network algorithm for performing Fisher's exact test in $\mathrm{r} \times \mathrm{c}$ contingency tables. J. Am. Stat. Assoc. 78:427-434.

Miller, R. H., M. T. Kuhn, H. D. Norman, and J. R. Wright. 2008. Death losses for lactating dairy cows in herds enrolled in dairy herd improvement test plans. J. Dairy Sci. 91:3710-3715.

NDAWB (National Dairy Animal Well-Being). 2008. Principles and guidelines. http://www.dairywellbeing.org/guidelines.php Accessed December 9, 2008.

Osteras, O., H. Solbu, A. O. Refsdal, T. Roalkvam, O. Filseth, and A. Minsaas. 2007. Results and evaluation of thirty years of health recordings in the Norwegian dairy cattle population. J. Dairy Sci. 90:4483-4497.

Santayana, G. 1917. Reason in Common Sense. Vol. 1. The Life of Reason, or, The Phases of Human Progress. Charles Scribner's Sons, New York, NY.

Severidt, J. A., D. J. Madden, G. L. Mason, F. B. Garry, and D. H. Gould. 2002. Dairy Cattle Necropsy Manual. Integrated Livestock Management, Colorado State University, Fort Collins, CO. http:// www.cvmbs.colostate.edu/ilm/proinfo/necropsy/notes/index.html Accessed Jan. 23, 2009.

Thomsen, P. T., and H. Houe. 2006. Dairy cow mortality: A review. Vet. Q. 28:122-129.

USDA. 2000a. Changes in the U.S. Feedlot Industry 1994-1999. Report from USDA:APHIS:VS, CEAH, National Animal Health Monitoring System, Fort Collins, CO.

USDA. 2000b. Part I: Baseline Reference of Feedlot Management Practices, 1999. Report from USDA:APHIS:VS, CEAH, National Animal Health Monitoring System. Fort Collins, CO.

USDA. 2007a. Dairy 2007, Part 1: Reference of Dairy Cattle Health and Management Practices in the United States, 2007. USDAAPHIS-VS, CEAH, Fort Collins, CO.

USDA. 2007b. Dairy 2007, Part II: Changes in the U.S. Dairy Cattle Industry, 1991-2007. USDA-APHIS-VS, CEAH, Fort Collins, CO.

Wagner, S. 2007. Necropsy techniques in cattle. Pages 203-204 in Proc. Fortieth Ann. Conf., Am. Assoc. Bovine Practitioners, Vancouver, British Columbia, Canada.

White, B. J. 2005. Field necropsy review. Page 67 in Proc. North Am. Vet. Conf. Large Animal, Vol. 19, Orlando, FL. B. J. White, ed. Eastern States Veterinary Association, Gainesville, FL.

Wildman, B. K., O. C. Schunicht, G. K. Jim, P. T. Guichon, C. W. Booker, and R. A. Tollens. 2000. The use of computer imaging technology to facilitate the capture of feedlot necropsy information. Can. Vet. J. 41:124-125. 